\title{
On Second Statistical Moments for Capricious Vague Perceptions*
}

\author{
Tokuo FUKUDA ${ }^{\dagger}$
}

\begin{abstract}
In this paper, the author investigates the statistical moments such as expectations and variances for a class of fuzzy random sets, where the fuzzy random set is considered as a model of the capricious vague perception of a crisp phenomenon or a crisp random phenomenon.

First, the class of fuzzy random sets, which has been proposed by author[1-3], where the vague perception of a crisp phenomenon fluctuates slightly but randomly by the state of a capricious person's mind, is refined and its expectation and variance are introduced.

Secondly, the refined class of fuzzy random sets is extended to the models for the capricious vague perceptions of crisp random phenomena, and their expectations and variances are investigated from the viewpoint of the multi-valued logic.
\end{abstract}

\section{Introduction}

Motivated by the importance for treating the data exhibiting both vagueness and randomness, fuzzy random sets or fuzzy random variables have been intensively investigated for a long time by many researchers with various definitions. For instance, the concept of fuzzy random variables obtained as vague linguistic observations of crisp random data was firstly presented by Kwakernaak[4], and investigated by e.g., Kruse[5,6]. On the other hand, Puri and Ralescu[7] defined firstly fuzzy random variables as the generalized random sets and discussed their statistical properties by many researchers, e.g., [8-10].

The purpose of this paper is to reconstruct fuzzy random sets as a model of the vague perceptions obtained from capricious persons, who may express the different feelings from one person to another with the same crisp observed values. For this purpose, a new class of fuzzy random sets is firstly introduced, considering that the vague perception of a crisp phenomenon fluctuates slightly but randomly due to the state of a capricious person's mind. The class of fuzzy random sets proposed in this paper is a revised one introduced in $[1,2]$. Secondly, the proposed fuzzy random set is generalized as the vague perception of a crisp random phenomenon. The expectations and the variances of the proposed fuzzy random sets are also investigated from the viewpoint of the multivalued logic proposed by Kwakernaak[4].

\footnotetext{
* Manuscript Received Date: May 13, 2013

$\dagger$ Faculty of Management, Otemon Gakuin University; 2-

1-15 Nishi-Ai, Ibaraki, Osaka 567-8502, JAPAN

Key Words: fuzzy random sets, capricious vague perception, multi-valued logic, expectation, variance.
}

\section{Fuzzy Random Sets as Models of Capricious Vague Perceptions of Crisp Phenomena}

In this section, we consider that the vague perception of a crisp phenomenon fluctuates slightly but randomly by the state of a capricious person's mind. Hence, the fuzzy set obtained as a vague perception of a crisp phenomenon may be some kind of 'fuzzy random set', i.e., it is a function of the generating point of some sample space. The proposed fuzzy random set is a revised one proposed by author last year[1-3].

[Example 1] Consider the situation that a person feels the temperature and the humidity, and he expresses his feeling like "dry", "comfortable", "sultry", "humid" and so on.

The linguistic data in the above example has two outstanding features. One is fuzziness due to the intrinsic vagueness of language, and another is the randomness caused by the capricious person's feelings, i.e., with same combination of the temperature and the humidity, some person will feel "humid" and another person will feel "comfortable". Furthermore, according to the person's physical condition, even same person sometimes feels "comfortable" and sometimes feels "sultry". This means that in this example the vague perception would be changed randomly along with its values. Then, the original point(fixed values), which is the object to be vaguely perceived by a person, may be given by

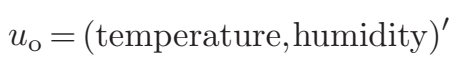

and the possible original point $\bar{u}$ of $u_{\mathrm{o}}$ should be a random variable in order to describe the capricious mind of person. Let the possible random original point $\bar{u}$ 
be defined on the probability space $\left(\bar{\Omega}, \overline{\mathcal{A}}, \bar{P}_{u_{\mathrm{o}}}\right)$, and consider here the subsets $\overline{\mathrm{A}}_{1}, \overline{\mathrm{A}}_{2}, \cdots, \overline{\mathrm{A}}_{M}$ of the sample space $\bar{\Omega}$ such as

$$
\begin{aligned}
& \overline{\mathrm{A}}_{1}=\{\omega \mid \text { the value of } \bar{u}(\omega) \\
& \text { represents "comfortable" }\} \\
& \overline{\mathrm{A}}_{2}=\{\omega \mid \text { the value of } \bar{u}(\omega) \text { represents "sultry" }\} \\
& \overline{\mathrm{A}}_{3}=\{\omega \mid \text { the value of } \bar{u}(\omega) \text { represents "humid" }\}
\end{aligned}
$$

where $\overline{\mathrm{A}}_{1}, \overline{\mathrm{A}}_{2}, \cdots, \overline{\mathrm{A}}_{M}$ are assumed to the elements of $\overline{\mathcal{A}}$ and they are elementary events satisfying the following properties:

$$
\left\{\begin{array}{l}
\bar{\Omega}=\overline{\mathrm{A}}_{1} \cup \overline{\mathrm{A}}_{2} \cup \cdots \cup \overline{\mathrm{A}}_{M}, \\
\overline{\mathrm{A}}_{i} \cap \overline{\mathrm{A}}_{j} \neq \emptyset \text { for any } i \neq j, \\
\sum_{i=1}^{M} \bar{P}_{u_{\mathrm{o}}}\left(\overline{\mathrm{A}}_{i}\right)=1, \\
\bar{P}_{u_{\mathrm{o}}}\left(\overline{\mathrm{A}}_{i}\right) \neq 0 \text { for any } i=1,2, \cdots, M
\end{array}\right.
$$

Then, we can see that the points in each subset $\overline{\mathrm{A}}_{i}(i=$ $1,2, \cdots, M)$ may not be discriminated. This implies that their details are lost due to the vague perception. Let $\overline{\mathcal{S}}$ be a sub $\sigma$-algebra of $\overline{\mathcal{A}}$ generated by $\overline{\mathrm{A}}_{1}, \overline{\mathrm{A}}_{2}, \cdots, \overline{\mathrm{A}}_{M}$ of non-distinguishable points caused by vague perceptions. Inspired by the idea given by Kwakernaak [4,11], define here an equivalence relation $\approx$ on $\bar{\Omega}$ as follows:

$$
\bar{\omega}_{1} \approx \bar{\omega}_{2}=\left[\forall \overline{\mathrm{A}} \in \overline{\mathcal{S}}: \bar{\omega}_{1} \in \overline{\mathrm{A}} \Leftrightarrow \bar{\omega}_{2} \in \overline{\mathrm{A}}\right]
$$

Let $c$ be the canonical projection from $\bar{\Omega}$ to the elementary sample space $\Omega=\left\{\omega_{1}, \omega_{2}, \cdots, \omega_{M}\right\}$ given as the quotient set of $\bar{\Omega}$ under $\approx$. Then, we can define the probability space $\left(\Omega, \mathcal{A}, P_{u_{\mathrm{o}}}\right)$ as the vague perception image, where

$$
\Omega=\bar{\Omega} \approx=\left\{\omega_{1}, \omega_{2}, \cdots, \omega_{M}\right\}
$$

$\mathcal{A}$ is the $\sigma$-algebra generated by $c(\overline{\mathrm{A}}), \overline{\mathrm{A}} \in \overline{\mathcal{A}}$, i.e.,

$$
\mathcal{A}=\{c(\overline{\mathrm{A}}) \mid \overline{\mathrm{A}} \in \overline{\mathcal{A}}\}
$$

and

$$
P_{u_{\mathrm{o}}}(\mathrm{A})=\bar{P}_{u_{\mathrm{o}}}\left(c^{-1}(\mathrm{~A})\right) \quad \text { for } \mathrm{A} \in \mathcal{A}
$$

According to the framework mentioned in the above example, the elementary discrete fuzzy random set is defined as follows:

[Definition 1] Let $\left(\Omega, \mathcal{A}, P_{u_{\mathrm{o}}}\right)$ be an elementary probability space given by eq. (3), eq. (4) and eq. (5). Then, an elementary fuzzy random set as a vague perception of the original point $u_{\mathrm{o}} \in \mathbb{R}^{n}$ is defined as follows:

$$
\widetilde{U}\left(u_{\mathrm{o}}, \omega\right)=\left(\mathbb{R}^{n},\left[\widetilde{U}\left(u_{\mathrm{o}}, \omega\right)\right], s_{\widetilde{U}}\right) \in \mathbb{F}_{\mathrm{cc}}^{\mathrm{b}}\left(\mathbb{R}^{n}\right)
$$

with

$$
\left[\widetilde{U}\left(u_{\mathrm{o}}, \omega\right)\right]=\left\{\left[\widetilde{U}\left(u_{\mathrm{o}}, \omega\right)\right]_{\alpha} \mid \alpha \in \mathbb{I}\right\}
$$

where $\mathbb{I}=(0,1)$ and

$$
\begin{aligned}
s_{\widetilde{U}}(u)=\{u \text { in } \widetilde{U} \text { coincides } \\
\text { with the original point } \left.u_{\mathrm{o}}\right\}
\end{aligned}
$$

For the class of fuzzy sets denoted by $\mathbb{F}_{\mathrm{cc}}^{\mathrm{b}}\left(\mathbb{R}^{n}\right)$ in eq. (6), see[1,2]. Then, we can rewrite eq. (6) by

$$
\widetilde{U}\left(u_{\mathrm{o}}, \omega\right)=\sum_{i=1}^{M} \mathbf{1}_{\omega_{i}}(\omega) \cdot \widetilde{U}_{i}
$$

where $\mathbf{1}_{\omega_{i}}(\omega)$ is the characteristic function of $\omega_{i}$ given by

$$
\mathbf{1}_{\omega_{i}}(\omega)=\left\{\begin{array}{l}
1, \text { if } \omega=\omega_{i} \\
0, \text { otherwise }
\end{array}\right.
$$

and $\widetilde{U}_{i}$ is the fuzzy set given by the triple

$$
\widetilde{U}_{i}=\left(\mathbb{R}^{n},\left[\widetilde{U}_{i}\right], s_{\widetilde{U}_{i}}\right) \in \mathbb{F}_{\mathrm{cc}}^{\mathrm{b}}\left(\mathbb{R}^{n}\right)
$$

with

$$
\left[\widetilde{U}_{i}\right]=\left\{\left[\widetilde{U}_{i}\right]_{\alpha} \mid \alpha \in \mathbb{I}\right\} \quad \text { for } \quad i=1,2,3, \cdots, M
$$

The predicate $s_{\widetilde{U}_{i}}$ in eq. (11) is given through the proposition

$$
\begin{aligned}
& s_{\widetilde{U}_{i}}(u)=\left\{u \text { in } \widetilde{U}_{i}\right. \text { coincides with } \\
& \text { the original point } \left.u_{\mathrm{o}}\right\}
\end{aligned}
$$

and its truth value is given by its membership function defined by

$$
\left(\widetilde{U}_{i}\right)(u)=t\left(s_{\widetilde{U}_{i}}(u)\right)
$$

where $t(*)$ is the truth function of $*$ in the sense of multi-valued logic. Hence, we have the relation between $[\widetilde{U}]_{\alpha}$ and $\left[\widetilde{U}_{i}\right]_{\alpha}(i=1,2, \cdots, M)$ such that

$$
[\widetilde{U}]_{\alpha}=\sum_{i=1}^{M} \mathbf{1}_{\omega_{i}}(\omega) \cdot\left[\widetilde{U}_{i}\right]_{\alpha} \quad \text { for each } \alpha \in \mathbb{I}
$$

The measurability of $\widetilde{U}\left(u_{\mathrm{o}}, \omega\right)$ is given through its $\mathcal{A}$ $\mathcal{B}$ measurability, i.e.,

$$
\widetilde{U}^{-1}\left(u_{\mathrm{o}}, \cdot\right)(\mathrm{B}) \in \mathcal{A} \quad \text { for } \forall \mathrm{B} \in \mathcal{B}
$$

where $\mathcal{B}$ is a $\sigma$-algebra generated by the subsets of $\mathbb{F}=\left\{\widetilde{U}_{1}, \widetilde{U}_{2}, \cdots, \widetilde{U}_{M}\right\}$

\section{Expectation of $\widetilde{U}\left(u_{\mathrm{o}}, \omega\right)$}

Since $\widetilde{U}\left(u_{\mathrm{o}}, \omega\right)$ is some kind of the random quantity, it should be possible to consider its statistical moments such as its expectation, its variance and so on. First, we consider the expectation of $U\left(u_{\mathrm{o}}, \omega\right)$.

By the way, it may be natural to consider that 
the probabilistic information concerned with an $\overline{\mathcal{A}}$ measurable possible random original point $\bar{u}$ is also decayed due to the vague perception. Here, we consider that the conditional expectation $E_{u_{\mathrm{o}}}(\bar{u} \mid \overline{\mathcal{S}})$ concerned with $\overline{\mathcal{S}}$ may describe the information decay of $\bar{u}$ by vague perceptions ${ }^{1}$. Then, it can be shown that

$$
E_{u_{\mathrm{o}}}(\bar{u} \mid \overline{\mathcal{S}})(\bar{\omega})=u_{i}(\text { some const.) }
$$

for all $\bar{\omega} \in \overline{\mathrm{A}}_{i} \in \overline{\mathcal{S}} \subset \overline{\mathcal{A}}$ at each $i=1,2, \cdots, M$. Then, it follows

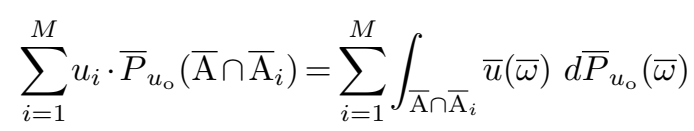

for $\forall \overline{\mathrm{A}} \in \overline{\mathcal{S}}$. Hence, by setting $\overline{\mathrm{A}}=\overline{\mathrm{A}}_{i}$, we have

$$
u_{i}=\frac{\int_{\overline{\mathrm{A}}_{i}} \bar{u}(\bar{\omega}) d \bar{P}_{u_{\mathrm{o}}}(\bar{\omega})}{\bar{P}_{u_{\mathrm{o}}}\left(\overline{\mathrm{A}}_{i}\right)}
$$

for each $i=1,2, \cdots, M$. Hence, we have

$$
E_{u_{\mathrm{o}}}(\bar{u} \mid \overline{\mathcal{S}})(\bar{\omega})=\sum_{i=1}^{M} \mathbf{1}_{\overline{\mathrm{A}}_{i}}(\bar{\omega}) \cdot u_{i}
$$

Then, we can define the random variable on $\left(\Omega, \mathcal{A}, P_{u_{\mathrm{o}}}\right)$ by

$$
u\left(\omega_{i}\right)=u_{i}=E_{u_{\mathrm{o}}}(\bar{u} \mid \overline{\mathcal{S}})(\bar{\omega}) \quad \text { for any } \bar{\omega} \in c^{-1}\left(\omega_{i}\right)
$$

at each $i=1,2, \cdots, M$.

With the reason mentioned above and in order to consider the expectation of an elementary fuzzy random set, we restrict hereafter the admissible class $\mathfrak{A}$ of the possible random original points to integrable $\mathcal{A}$-measurable ones given as follows:

$$
\begin{aligned}
\mathfrak{A}= & \left\{u \mid u(\omega)=\sum_{i=1}^{M} \mathbf{1}_{\omega_{i}}(\omega) \cdot u_{i},\right. \\
& \text { and } \left.u_{i} \in \mathbb{R}^{n} \text { for each } i=1,2, \cdots, M\right\}
\end{aligned}
$$

[Definition 2] Let $\widetilde{U}\left(u_{\mathrm{o}}, \omega\right) \in \mathbb{F}_{\mathrm{cc}}^{\mathrm{b}}\left(\mathbb{R}^{n}\right)$ be an elementary fuzzy random set. Then, the expectation of $\widetilde{U}$ is defined by

$$
\begin{gathered}
\mathcal{E}_{u_{\mathrm{o}}}[\widetilde{U}]=\left(\mathbb{R}^{n},\left[\mathcal{E}_{u_{\mathrm{o}}}[\widetilde{U}]\right], s_{\mathcal{E}_{u_{\mathrm{o}}}[\widetilde{U}]}\right) \\
=\sum_{i=1}^{M} \widetilde{U}_{i} \cdot P_{u_{\mathrm{o}}}\left(\omega_{i}\right)
\end{gathered}
$$

with its set representation given by

$$
\left[\mathcal{E}_{u_{\mathrm{o}}}[\widetilde{U}]\right]=\left\{\left[\mathcal{E}_{u_{\mathrm{o}}}[\widetilde{U}]\right]_{\alpha} \mid \alpha \in \mathbb{I}\right\}
$$

and

\footnotetext{
${ }^{1}$ Although there are no consideration on the capricious vague perception of crisp phenomena, we have developed a similar discussion in[12].
}

$$
\left[\mathcal{E}_{u_{\mathrm{o}}}[\widetilde{U}]\right]_{\alpha}=E_{u_{\mathrm{o}}}\left[[\widetilde{U}]_{\alpha}\right]=\sum_{i=1}^{M}\left[\widetilde{U}_{i}\right]_{\alpha} \cdot P_{u_{\mathrm{o}}}\left(\omega_{i}\right)
$$

where $s_{\mathcal{E}_{u_{\mathrm{o}}}[\widetilde{U}]}$ in eq. (18) is the predicate associated with the proposition given by

$$
\begin{aligned}
s_{\mathcal{E}_{u_{\mathrm{o}}[\widetilde{U}]}}(x)=\left\{\left(x=E_{u_{\mathrm{o}}}(u)\right)\right. \text { and } \\
\\
\left.\quad\left(u=u_{\mathrm{o}} \text { for some } u \in \mathfrak{A}\right)\right\}
\end{aligned}
$$

with

$$
E_{u_{\mathrm{o}}}(u)=\sum_{i=1}^{M} u_{i} \cdot P_{u_{\mathrm{o}}}\left(\omega_{i}\right)
$$

where $u=\sum_{i=1}^{M} \mathbf{1}_{\omega_{i}}(\omega) \cdot u_{i}$ is an element of $\mathfrak{A}$, and it should be noted that the probability $P_{u_{\mathrm{o}}}\left(\omega_{i}\right)$ depends on the value of the original point $u_{\mathrm{o}}$.

Then, we have the following proposition(see[1,2]).

[Proposition 1] Let $\widetilde{U}\left(u_{\mathrm{o}}, \omega\right)$ be an n-dimensional elementary random set, i.e., $\widetilde{U} \in \mathbb{F}_{\mathrm{cc}}^{\mathrm{b}}\left(\mathbb{R}^{n}\right)$. Then, there exists a fuzzy set such that

$$
\mathcal{E}_{u_{\mathrm{o}}}[\widetilde{U}]=\sum_{i=1}^{M} \widetilde{U}_{i} \cdot P_{u_{\mathrm{o}}}(\omega) \in \mathbb{F}_{\mathrm{cc}}^{\mathrm{b}}\left(\mathbb{R}^{n}\right)
$$

(Remark 1) Let $\mathfrak{A}_{\alpha}$ be the selection set defined by

$$
\mathfrak{A}_{\alpha}=\left\{u \mid u(\omega) \in \mathfrak{A} \quad \text { and } \quad u(\omega) \in\left[\widetilde{U}\left(u_{\mathrm{o}}, \omega\right)\right]_{\alpha}\right\}(2
$$

Then, the element of the set representation of $\mathcal{E}_{u_{\mathrm{o}}}[\tilde{U}]$ given by eq. (20) is rewritten as follows:

$$
\left[\mathcal{E}_{u_{\mathrm{o}}}[\widetilde{U}]\right]_{\alpha}=E_{u_{\mathrm{o}}}\left[[\widetilde{U}]_{\alpha}\right]=\left\{E_{u_{\mathrm{o}}}(u) \mid u \in \mathfrak{A}_{\alpha}\right\}
$$

\section{Variance of $\widetilde{U}\left(u_{\mathrm{o}}, \omega\right)$}

Let $\widetilde{U}\left(u_{\mathrm{o}}, \omega\right) \in \mathbb{R}^{n}$ be an elementary fuzzy random set defined in Definition 1, i.e., $\widetilde{U}\left(u_{\mathrm{o}}, \omega\right)$ is the fuzzy random set as the model of a capricious vague perception of some crisp non-random phenomenon. Consider here the proposition given by

$$
\begin{array}{r}
s_{\mathcal{V}_{m, u_{\mathrm{o}}} \widetilde{U}}(x)=\left\{\left(x=\operatorname{var}_{u_{\mathrm{o}}} u\right)\right. \\
\text { and } \left.\left(u=u_{\mathrm{o}} \text { for some } u \in \mathfrak{A}\right)\right\}
\end{array}
$$

with

$$
\operatorname{var}_{u_{\mathrm{o}}} u=E_{u_{\mathrm{o}}}\left(u-E_{u_{\mathrm{o}}}(u)\right)\left(u-E_{u_{\mathrm{o}}}(u)\right)^{\prime}
$$

where $E_{u_{\mathrm{o}}}(u)$ is the expectation of $u$. Then, the above proposition may be given by a composite proposition such as

$$
\begin{aligned}
s_{\mathcal{V}_{m, u_{\mathrm{o}}} \widetilde{U}}(x)=\bigvee_{u \in \mathfrak{A}}\{ & \left(x=\operatorname{var}_{u_{\mathrm{o}}} u\right) \\
& \left.\wedge\left(\bigwedge_{i=1}^{M}\left(u_{i}=u_{\mathrm{o}}\right)\right)\right\}
\end{aligned}
$$


Therefore, applying the fuzzy logic(see e.g., $[4,5,12])$, the truth value of $s_{\mathcal{V}_{m, u_{\mathrm{o}}}} \widetilde{U}(x)$ is given by

$$
\begin{aligned}
& t\left(s_{\mathcal{V}_{m, u_{\mathrm{o}}} \widetilde{U}}(x)\right) \\
& =\sup _{u \in \mathfrak{A}}\left\{\min _{i=1,2, \cdots, M}\left\{\left(\widetilde{U}_{i}\right)(u)\right\} \mid x=\operatorname{var}_{u_{\mathrm{o}}} u\right\}
\end{aligned}
$$

where $\left(\widetilde{U}_{i}\right)(u)$ is given by eq. (14). Then, using the similar procedures as those shown in[13-15], we can confirm the following property holds:

[Proposition 2] Let $\widetilde{U}\left(u_{\mathrm{o}}, \omega\right)$ be an elementary fuzzy random set. Then,

$$
\begin{aligned}
\left\{x \mid t\left(s_{\mathcal{V}_{m, u_{\mathrm{o}}} \widetilde{U}}(x)\right)>\alpha\right\} \subseteq \mathrm{V}_{m, u_{\mathrm{o}}}[\widetilde{U}]_{\alpha} \\
\quad \subseteq\left\{x \mid t\left(s_{\mathcal{V}_{m, u_{\mathrm{o}}} \widetilde{U}}(x)\right) \geq \alpha\right\}
\end{aligned}
$$

for any $\alpha \in \mathbb{I}$, where $\mathrm{V}_{m, u_{\mathrm{o}}}[\tilde{U}]_{\alpha}$ is given by

$$
\mathrm{V}_{m, u_{\mathrm{o}}}[\widetilde{U}]_{\alpha}=\left\{\operatorname{var}_{u_{\mathrm{o}}} u \mid u \in \mathfrak{A}_{\alpha}\right\}
$$

and $\mathfrak{A}_{\alpha}$ in eq. (31) is given by eq. (24).

(Proof) From the definition of the set representation of fuzzy sets(see e.g.[1,2,5]), we have

$$
L_{\alpha} \widetilde{U}_{i} \subseteq\left[\widetilde{U}_{i}\right]_{\alpha} \subseteq L_{\bar{\alpha}} \widetilde{U}_{i}
$$

Then, it follows that

$$
\mathbf{1}_{\omega_{i}}(\omega) \cdot L_{\alpha} \widetilde{U}_{i} \subseteq \mathbf{1}_{\omega_{i}}(\omega) \cdot\left[\widetilde{U}_{i}\right]_{\alpha} \subseteq \mathbf{1}_{\omega_{i}}(\omega) \cdot L_{\bar{\alpha}} \widetilde{U}_{i}
$$

for each $\alpha \in \mathbb{I}$ and $\omega \in \Omega$. Furthermore, it can be shown that

$$
L_{\alpha} \widetilde{U}=\sum_{i=1}^{M} \mathbf{1}_{\omega_{i}}(\omega) \cdot L_{\alpha} \widetilde{U}_{i}
$$

and

$$
L_{\bar{\alpha}} \widetilde{U}=\sum_{i=1}^{M} \mathbf{1}_{\omega_{i}}(\omega) \cdot L_{\bar{\alpha}} \widetilde{U}_{i}
$$

Hence, from eq. (15), eq. (32), eq. (33) and eq. (34) it follows that

$$
L_{\alpha} \widetilde{U} \subseteq[\widetilde{U}]_{\alpha} \subseteq L_{\bar{\alpha}} \widetilde{U}
$$

for each $\alpha \in \mathbb{I}$, and we have

$$
\mathrm{V}_{m, u_{\mathrm{o}}}\left(L_{\alpha} \widetilde{U}\right) \subseteq \mathrm{V}_{m, u_{\mathrm{o}}}[\widetilde{U}]_{\alpha} \subseteq \mathrm{V}_{m, u_{\mathrm{o}}}\left(L_{\bar{\alpha}} \widetilde{U}\right)
$$

for each $\alpha \in \mathbb{I}$, where

$$
\mathrm{V}_{m, u_{\mathrm{o}}}\left(L_{\alpha} \widetilde{U}\right)=\left\{\operatorname{var}_{u_{\mathrm{o}}} u \mid u \in \mathfrak{A} \text { and } u \in L_{\alpha} \widetilde{U}\right\}
$$

and

$$
\mathrm{V}_{m, u_{\mathrm{o}}}\left(L_{\bar{\alpha}} \widetilde{U}\right)=\left\{\operatorname{var}_{u_{\mathrm{o}}} u \mid u \in \mathfrak{A} \text { and } u \in L_{\bar{\alpha}} \widetilde{U}\right\}
$$

(i) If there exists a vector $\zeta$ such that

$$
\zeta \in\left\{x \mid t\left(s_{\mathcal{V}_{m, u_{\mathrm{o}}} \widetilde{U}}(x)\right)>\alpha\right\}
$$

$$
=\left\{x \mid \sup _{u \in \mathfrak{A}}\left\{\min _{i=1,2, \cdots, M}\left\{\left(\widetilde{U}_{i}\right)\left(u_{i}\right)\right\} \mid x=\operatorname{var}_{u_{\mathrm{o}}} u\right\}>\alpha\right\}
$$

there is some element $\xi$ of $\mathfrak{A}$ satisfying

$$
\zeta=\operatorname{var}_{u_{\mathrm{o}}} \xi=\sum_{i=1}^{M}\left(\xi_{i}-E_{u_{\mathrm{o}}}(\xi)\right)\left(\xi_{i}-E_{u_{\mathrm{o}}}(\xi)\right)^{\prime} \cdot P_{u_{\mathrm{o}}}\left(\omega_{i}\right)
$$

and

$$
\left(\widetilde{U}_{i}\right)\left(\xi_{i}\right)>\alpha
$$

for any $i=1,2, \cdots, M$, which means

$$
\xi_{i} \in L_{\alpha} \widetilde{U}_{i}
$$

Hence, we have

$$
\zeta \in \mathrm{V}_{m, u_{\mathrm{o}}}\left(L_{\alpha} \widetilde{U}\right)
$$

or equivalently

$$
\left\{x \mid t\left(s_{\mathcal{V}_{m, u_{\mathrm{o}}} \widetilde{U}}(x)\right)>\alpha\right\} \subseteq \mathrm{V}_{m, u_{\mathrm{o}}}\left(L_{\alpha} \widetilde{U}\right)
$$

for any $\alpha \in \mathbb{I}$.

(ii) If there exists $\zeta$ such that

$$
\zeta \in \mathrm{V}_{m, u_{\mathrm{o}}}\left(L_{\bar{\alpha}} \widetilde{U}\right)
$$

there is some element $\xi \in \mathfrak{A}$ satisfying $\zeta=\operatorname{var}_{u_{\mathrm{o}}} \xi$ and

$$
\left(\widetilde{U}_{i}\right)\left(\xi_{i}\right) \geq \alpha \quad \text { for any } i=1,2, \cdots, M
$$

which means

$$
\min _{i=1,2, \cdots, M}\left\{\left(\widetilde{U}_{i}\right)\left(\xi_{i}\right)\right\} \geq \alpha
$$

and hence it follows that

$$
\begin{aligned}
\zeta & \in\left\{x \mid \sup _{\xi \in \mathfrak{A}}\left\{\min _{i=1,2, \cdots, M}\left\{\widetilde{U}_{i}\left(\xi_{i}\right)\right\} \mid x=\operatorname{var}_{u_{\mathrm{o}}} \xi\right\} \geq \alpha\right\} \\
& =\left\{x \mid t\left(s_{\mathcal{V}_{m, u_{\mathrm{o}}} \widetilde{U}}(x)\right) \geq \alpha\right\}
\end{aligned}
$$

or equivalently

$$
\mathrm{V}_{m, u_{\mathrm{o}}}\left(L_{\bar{\alpha}} \widetilde{U}\right) \subseteq\left\{x \mid t\left(s_{\mathcal{V}_{m, u_{\mathrm{o}}} \widetilde{U}}(x)\right) \geq \alpha\right\}
$$

for $\alpha \in \mathbb{I}$.

(iii) From eq. (36), eq. (40) and eq. (41), we have eq. (30).

Therefore, the following definition of the variance of an elementary fuzzy random set $\widetilde{U}\left(u_{\mathrm{o}}, \omega\right)$ may be reasonable:

[Definition 3] Let $\widetilde{U}\left(u_{\mathrm{o}}, \omega\right) \in \mathbb{F}_{\mathrm{cc}}^{\mathrm{b}}\left(\mathbb{R}^{n}\right)$ be an elementary fuzzy random set defined by Definition 1. Then, the variance of $\widetilde{U}$ is given by

$$
\mathcal{V}_{m, u_{\mathrm{o}}} \widetilde{U}=\left(\mathbb{R}^{n \times n},\left[\mathcal{V}_{m, u_{\mathrm{o}}} \widetilde{U}\right], s_{\mathcal{V}_{m, u_{\mathrm{o}}} \widetilde{U}}\right)
$$

with its set representation given by 


$$
\left[\mathcal{V}_{m, u_{\mathrm{o}}} \tilde{U}\right]=\left\{\mathrm{V}_{m, u_{\mathrm{o}}}[\widetilde{U}]_{\alpha} \mid \alpha \in \mathbb{I}\right\}
$$

where $\mathrm{V}_{m, u_{\mathrm{o}}}[\widetilde{U}]_{\alpha}$ is given by eq. (31). The predicate $s_{\mathcal{V}_{m, u_{0}} \widetilde{U}}$ in eq. (42) is given through eq. (26).

The following corollary is obtained immediately from eq. (36), eq. (40) and eq. (41) in the proof of Proposition 2.

[Corollary 1] Let $\widetilde{U}\left(u_{\mathrm{o}}, \omega\right)$ be an elementary fuzzy random set. Then we have

$$
\begin{aligned}
& L_{\alpha}\left(\mathcal{V}_{m, u_{\mathrm{o}}} \widetilde{U}\right) \subseteq \mathrm{V}_{m, u_{\mathrm{o}}}\left(L_{\alpha} \widetilde{U}\right) \subseteq\left[\mathcal{V}_{m, u_{\mathrm{o}}} \widetilde{U}\right]_{\alpha} \\
& =\mathrm{V}_{m, u_{\mathrm{o}}}[\widetilde{U}]_{\alpha} \subseteq \mathrm{V}_{m, u_{\mathrm{o}}}\left(L_{\bar{\alpha}} \widetilde{U}\right) \subseteq L_{\bar{\alpha}}\left(\mathcal{V}_{m, u_{\mathrm{o}}} \widetilde{U}\right)
\end{aligned}
$$

for any $\alpha \in \mathbb{I}$, where

$$
L_{\alpha}\left(\mathcal{V}_{m, u_{o}} \widetilde{U}\right)=\left\{x \mid t\left(s_{\mathcal{V}_{m, u_{o}} \widetilde{U}}(x)\right)>\alpha\right\}
$$

and

$$
L_{\bar{\alpha}}\left(\mathcal{V}_{m, u_{o}} \widetilde{U}\right)=\left\{x \mid t\left(s_{\mathcal{V}_{m, u_{o}} \widetilde{U}}(x)\right) \geq \alpha\right\}
$$

\section{Fuzzy Random Sets as Models of Capricious Vague Perceptions of Random Phenomena}

There are many crisp phenomena that are perceived vaguely as mentioned in Sec. 2., and also there are many crisp phenomena which are themselves randomly changed. For instance, the temperature and the humidity of the example in Sec. 2. may fluctuate randomly due to the weather conditions. Therefore, in order to consider the vague perceptions of random phenomena, two types of randomness should be considered, one of which is the randomness due to the capricious person's feelings and another of which is the randomness of the phenomena themselves.

The class of fuzzy random sets investigated in this section is an extended one of that in Sec. 2., and hence it is also revised one of that proposed in $[1,2]$. Let $\left(\Omega_{1}, \mathcal{A}_{1}, P_{1}\right)$ be an elementary probability space describing the randomness of capricious persons' minds defined as $(\Omega, \mathcal{A}, P)$ in Definition 1, and let $\left(\Omega_{2}, \mathcal{A}_{2}, P_{2}\right)$ be a probability space, on which an original random point $u_{\mathrm{o}} \in \mathbb{R}^{n}$ as the model of a random phenomenon is defined. Then, the extended fuzzy random set as a capricious vague perception of the original random point $u_{\mathrm{o}}$ is defined on $(\Omega, \mathcal{A}, P)=\left(\Omega_{1} \times \Omega_{2}, \mathcal{A}_{1} \otimes\right.$ $\left.\mathcal{A}_{2}, P_{1} \times P_{2}\right)$ and given as follows:

[Definition 4] An extended fuzzy random set $\widetilde{U}(\omega)$ on $(\Omega, \mathcal{A}, P)$ obtained as the capricious vague perception of an original random point $u_{\mathrm{o}}\left(\omega^{(2)}\right)$ on $\left(\Omega_{2}, \mathcal{A}_{2}, P_{2}\right)$ is defined by

$$
\widetilde{U}(\omega)=\left(\mathbb{R}^{n},[\widetilde{U}(\omega)], s_{\widetilde{U}}\right) \in \mathbb{F}_{\mathrm{cc}}^{\mathrm{b}}\left(\mathbb{R}^{n}\right)
$$

with

$$
[\widetilde{U}(\omega)]=\left\{[\widetilde{U}(\omega)]_{\alpha} \mid \alpha \in \mathbb{I}\right\}
$$

sition such as

$$
\begin{array}{r}
s_{\widetilde{U}}(u)=\{u \text { in } \widetilde{U} \text { coincides with the original } \\
\text { random point } \left.u_{\mathrm{o}}\right\}
\end{array}
$$

Then, we can rewrite $\widetilde{U}(\omega)$ in eq. (47) by

$$
\widetilde{U}(\omega)=\sum_{i=1}^{M} \mathbf{1}_{\omega_{i}^{(1)}}(\omega) \cdot \widetilde{U}_{i}
$$

where $\left\{\widetilde{U}_{i} ; i=1,2, \cdots, M\right\}$ is a collection of fuzzy random sets given by eq. (11), and

$$
\mathbf{1}_{\omega_{i}^{(1)}}(\omega)=\left\{\begin{array}{l}
1, \text { if } \omega \in\left\{\omega_{i}^{(1)}\right\} \times \Omega_{2} \\
0, \text { otherwise }
\end{array}\right.
$$

The measurability of $\widetilde{U}$ is given through

$$
\widetilde{U}^{-1}(\mathrm{~B}) \in \mathcal{A}=\mathcal{A}_{1} \otimes \mathcal{A}_{2} \quad \text { for any } \mathrm{B} \in \mathcal{B}
$$

where $\mathcal{B}$ is given in Definition $\mathbf{1}$, and the admissible class of possible original random points $\mathfrak{A}_{e}$ is assumed to be given by

$$
\begin{aligned}
\mathfrak{A}_{e}= & \{u \mid \text { integrable random variables on }(\Omega, \mathcal{A}, P)\} \\
= & \left\{u \mid u(\omega)=\sum_{i=1}^{M} \mathbf{1}_{\omega_{i}^{(1)}}(\omega) \cdot \xi\left(\omega^{(2)}\right) ;\right. \\
& \left.\xi \text { is the integrable on }\left(\Omega_{2}, \mathcal{A}_{2}, P_{2}\right)\right\}
\end{aligned}
$$

\section{Expectation of $\widetilde{U}(\omega)$}

As described in Sec. 5., a capricious vague perception of the original random point $u_{\mathrm{o}}\left(\omega^{(2)}\right)$ is modeled by the fuzzy random set $\widetilde{U}(\omega)$ given by eq. (47).

Applying the similar procedure as that for the fuzzy random set proposed in[1,2], we can show that the expectation of a fuzzy random set $\widetilde{U}$ may be given as follows:

[Definition 5] Let $\widetilde{U}=\left(\mathbb{R}^{n},[\widetilde{U}], s_{\widetilde{U}}\right)$ be an extended fuzzy random set given by eq. (47). Then, the expectation of $\widetilde{U}$ is given by

$$
\mathcal{E}[\widetilde{U}]=\left(\mathbb{R}^{n},[\mathcal{E}[\widetilde{U}]], s_{\mathcal{E}[\widetilde{U}]}\right)
$$

with

$$
[\mathcal{E}[\widetilde{U}]]=\left\{E\left[[\widetilde{U}]_{\alpha}\right] \mid \alpha \in I\right\}
$$

where $s_{\mathcal{E}[\widetilde{U}]}$ is the predicate associated with the proposition given by

$$
\begin{aligned}
& s_{\mathcal{E}[\widetilde{U}]}(x)=\{x \text { coincides with } \\
&\text { the expectation of } \left.u_{\mathrm{o}}\right\}
\end{aligned}
$$

and $[\mathcal{E}[\widetilde{U}]]$ is the set representation of $\mathcal{E}[\widetilde{U}]$ given through

where $s_{\widetilde{U}}$ is the predicate associated with the propo- 


$$
E\left[[\widetilde{U}]_{\alpha}\right]=\int \sum_{i=1}^{M} \mathbf{1}_{\omega_{i}^{(1)}}(\omega) \cdot\left[\widetilde{U}_{i}\right]_{\alpha} P\left(\omega_{i}^{(1)}, d \omega^{(2)}\right)
$$

Let here $\mathcal{S}$ be the sub $\sigma$-algebra of $\mathcal{A}$ consisting all cylinder sets of the form $A=\Omega_{1} \times A^{(2)}$ with $A^{(2)} \in$ $\mathcal{A}_{2}$. Then, the conditional expectation of $\widetilde{U}$ concerned with $\mathcal{S}$ should be given as follows:

$$
\mathcal{E}[\widetilde{U} \mid \mathcal{S}]=\left(\mathbb{R}^{n},[\mathcal{E}[\widetilde{U} \mid \mathcal{S}]], s_{\mathcal{E}[\widetilde{U} \mid \mathcal{S}]}\right)
$$

with

$$
[\mathcal{E}[\widetilde{U} \mid \mathcal{S}]]=\left\{E\left[[\widetilde{U}]_{\alpha} \mid \mathcal{S}\right] \mid \alpha \in I\right\}
$$

[Proposition 3] Let $\widetilde{U}=\left(\mathbb{R}^{n},[\widetilde{U}], s_{\widetilde{U}}\right)$ be an extended fuzzy random set given by eq. (47). Then, it follows

$$
\mathcal{E}[\widetilde{U}]=\mathcal{E}[\mathcal{E}[\widetilde{U} \mid \mathcal{S}]]
$$

where $\mathcal{E}[\widetilde{U} \mid \mathcal{S}]$ defined by eq. (58) is given by

$$
\mathcal{E}[\widetilde{U} \mid \mathcal{S}]=\sum_{i=1}^{M} \widetilde{U}_{i} \cdot P\left(\omega_{i}^{(1)} \mid \mathcal{S}\right)
$$

(Remark 2) Let $\mathfrak{A}_{e, \alpha}$ be the selection set defined by

$$
\mathfrak{A}_{e, \alpha}=\left\{u \mid u(\omega) \in \mathfrak{A}_{e} \operatorname{and} u(\omega) \in[\widetilde{U}(\omega)]_{\alpha}\right\}
$$

Then, the element of the set representation of $\mathcal{E}[\widetilde{U}]$ given by eq. (57) is rewritten as follows:

$$
[\mathcal{E}[\widetilde{U}]]_{\alpha}=E\left[[\widetilde{U}]_{\alpha}\right]=\left\{E(u) \mid u \in \mathfrak{A}_{e, \alpha}\right\}
$$

where $E(u)$ is the expectation of $u$ given by $E(u)=$ $\int u(\omega) d P(\omega)$.

\section{Variance of $\widetilde{U}(\omega)$}

Let $\widetilde{U}(\omega) \in \mathbb{R}^{n}$ be an extended fuzzy random set defined in Definition 4, i.e., $\widetilde{U}(\omega)$ is the fuzzy random set as the model of a capricious vague perception on some crisp random phenomenon. Consider here the proposition given by

$$
s_{\mathcal{V}_{m} \widetilde{U}}(x)=\left\{x \text { coincides with var. } u_{\mathrm{o}}\right\}
$$

with var. $u_{\mathrm{o}}=E\left(u_{\mathrm{o}}-E\left(u_{\mathrm{o}}\right)\right)\left(u_{\mathrm{o}}-E\left(u_{\mathrm{o}}\right)\right)^{\prime}$. Then, the above proposition may be given by a composite proposition such as

$$
\begin{aligned}
& s_{\mathcal{V}_{m} \widetilde{U}}(x)=\bigvee_{u \in \mathfrak{A}_{e}}\{(x=\text { var. } u) \\
& \left.\wedge\left(\bigwedge_{\substack{i=1 \\
P_{1}\left(\omega_{i}^{(1)}\right)>0}}^{M} \bigwedge_{\omega^{(2)} \in \Omega_{2} \backslash \mathrm{N}_{0}} s_{\widetilde{U}_{i}}\left(\xi\left(\omega^{(2)}\right)\right)\right)\right\}
\end{aligned}
$$

Therefore, applying the fuzzy logic, the truth value of $s_{\mathcal{V}_{m} \widetilde{U}}(x)$ is given by

$$
\begin{aligned}
& t\left(s_{\mathcal{V}_{m} \widetilde{U}}(x)\right) \\
= & \sup _{u \in \mathfrak{A}_{e}}\left\{\min _{\substack{i=1,2, \cdots, M \\
P_{1}\left(\omega_{i}^{(1)}\right)>0}}\left\{\operatorname{eins}_{\omega(2) \in \Omega_{2}}\left(\widetilde{U}_{i}\right)(\xi)\right\} \mid x=\text { var. } u\right\}(66)
\end{aligned}
$$

where $\left(\widetilde{U}_{i}\right)(\xi)$ is given by eq. (14). Then, using the similar procedure as that shown in Sec. 4., we can confirm the following property holds:

[Proposition 4] Let $\widetilde{U}(\omega)$ be an extended fuzzy random set. Then,

$$
\begin{aligned}
\left\{x \mid t\left(s_{\mathcal{V}_{m} \widetilde{U}}(x)\right)>\alpha\right\} & \subseteq \mathrm{V}_{m}[\widetilde{U}]_{\alpha} \\
& \subseteq\left\{x \mid t\left(s_{\mathcal{V}_{m} \widetilde{U}}(x)\right) \geq \alpha\right\}
\end{aligned}
$$

for any $\alpha \in \mathbb{I}$, where $\mathrm{V}_{m}[\tilde{U}]_{\alpha}$ is given by

$$
\mathrm{V}_{m}[\widetilde{U}]_{\alpha}=\left\{\operatorname{var} . u \mid u \in \mathfrak{A}_{e, \alpha}\right\}
$$

and $\mathfrak{A}_{e, \alpha}$ in eq. (68) is given by eq. (53).

Therefore, the following definition of the variance of an extended fuzzy random set $\widetilde{U}(\omega)$ may be reasonable:

[Definition 6] Let $\widetilde{U}(\omega) \in \mathbb{F}_{\mathrm{cc}}^{\mathrm{b}}\left(\mathbb{R}^{n}\right)$ be an extended fuzzy random set defined in Definition 4. Then, the variance of $\widetilde{U}$ is given by

$$
\mathcal{V}_{m} \widetilde{U}=\left(\mathbb{R}^{n \times n},\left[\mathcal{V}_{m} \widetilde{U}\right], s_{\mathcal{V}_{m} \widetilde{U}}\right)
$$

with its set representation given by

$$
\left[\mathcal{V}_{m} \widetilde{U}\right]=\left\{\mathrm{V}_{m}[\widetilde{U}]_{\alpha} \mid \alpha \in \mathbb{I}\right\}
$$

and $\mathrm{V}_{m}[\widetilde{U}]_{\alpha}$ is given by eq. (68). The predicate $s_{\mathcal{V}_{m} \widetilde{U}}$ in eq. (69) is given through eq. (64).

The following corollary is obtained by the similar procedure as that for Corollary $\mathbf{1}$.

[Corollary 2] Let $\widetilde{U}(\omega)$ be an extended fuzzy random set obtained as a capricious vague perception of the random phenomenon. Then we have

$$
\begin{aligned}
& L_{\alpha}\left(\mathcal{V}_{m} \widetilde{U}\right) \subseteq \mathrm{V}_{m}\left(L_{\alpha} \widetilde{U}\right) \subseteq\left[\mathcal{V}_{m} \widetilde{U}\right]_{\alpha} \\
& =\mathrm{V}_{m}[\widetilde{U}]_{\alpha} \subseteq \mathrm{V}_{m}\left(L_{\bar{\alpha}} \widetilde{U}\right) \subseteq L_{\bar{\alpha}}\left(\mathcal{V}_{m} \widetilde{U}\right)
\end{aligned}
$$

for any $\alpha \in \mathbb{I}$, where

$$
L_{\alpha}\left(\mathcal{V}_{m} \widetilde{U}\right)=\left\{x \mid t\left(s_{\mathcal{V}_{m} \widetilde{U}}(x)\right)>\alpha\right\}
$$

and

$$
L_{\bar{\alpha}}\left(\mathcal{V}_{m} \widetilde{U}\right)=\left\{x \mid t\left(s_{\mathcal{V}_{m} \widetilde{U}}(x)\right) \geq \alpha\right\}
$$

\section{Concluding Remarks}

In this paper, the author has introduced a probabilistic approach for representing the vague perception obtained from capricious persons, which is the revised version of $[1,2]$, and has investigated the statistical moments such as the expectation and the variance for a class of fuzzy random sets as models of capricious 
vague perceptions of crisp non-random phenomena.

Secondly, the introduced class of fuzzy random sets have been extended for the capricious vague perception of crisp random phenomena, and the expectation and the variance for a class of the extended fuzzy random sets have been also investigated from the viewpoint of the multi-valued logic.

Both for an elementary fuzzy random set $\widetilde{U}\left(u_{\mathrm{o}}, \omega\right)$ and an extended one $\widetilde{U}(\omega)$, another notion of variances obtained from Frèchet approach can be defined [15-17]. For instance, the variance of an elementary fuzzy random set $\widetilde{U}\left(u_{\mathrm{o}}, \omega\right)$ by Fréchet approach is given by

$$
\begin{aligned}
\mathrm{V}_{F, u_{\mathrm{o}}} \widetilde{U} & =E\left(\rho_{2}^{2}\left(\widetilde{U}, \mathcal{E}_{u_{\mathrm{o}}}[\widetilde{U}]\right)\right) \\
& =\inf \left\{E\left(\rho_{2}^{2}(\widetilde{U}, \widetilde{V})\right) \mid \widetilde{V} \in \mathbb{F}_{\mathrm{cc}}^{\mathrm{b}}\left(\mathbb{R}^{n}\right)\right\}
\end{aligned}
$$

provided that there exists $\mathcal{E}_{u_{\mathrm{o}}}[\widetilde{U}] \in \mathbb{F}_{\mathrm{cc}}^{\mathrm{b}}\left(\left[\mathbb{R}^{n}\right)\right.$, where $\rho_{2}$ is a metric of $\mathbb{F}_{\mathrm{cc}}^{\mathrm{b}}\left(\mathbb{R}^{n}\right)[2]$. Furthermore, the expectation of $\widetilde{U}\left(u_{\mathrm{o}}, \omega\right)$ by Fréchet approach is given by

$$
\mathcal{E}_{u_{\mathrm{o}}}[\widetilde{U}]=\arg \left\{\inf \left\{E\left(\rho_{2}^{2}(\widetilde{U}, \widetilde{V})\right) \mid \widetilde{V} \in \mathbb{F}_{\mathrm{cc}}^{\mathrm{b}}\left(\mathbb{R}^{n}\right)\right\}\right\}
$$

which coincides with that given by eq. (18). The detailed aspect of this notion of variances for $\widetilde{U}\left(u_{\mathrm{o}}, \omega\right)$ and $\widetilde{U}(\omega)$ will be reported in the near future.

\section{References}

[1] T. Fukuda: Probabilistic approach for vague perceptions of complex phenomena; Proceedings of The 43rd ISCIE International Symposium on Stochastic Systems Theory and its Applications, pp.101-106 (2012)

[2] T. Fukuda: On a probabilistic approach for capricious vague perceptions of random phenomena; Transactions of ISCIE, Vol. 25, No. 12, pp.382-388 (2012)

[3] T. Fukuda: On statistical moments for capricious vague perceptions; Proceedings of The 44th ISCIE International Symposium on Stochastic Systems Theory and its Applications, 2013 (to appear)

[4] H. Kwakernaak: Fuzzy random variables-I: Definitions and theorems; Information Sciences, Vol. 15, pp.1-29 (1978)

[5] R. Kruse and K. D. Meyer: Statistics with Vague Data, D. Reidel Publishing Company (1987)

[6] R. Kruse, E. Schwecke and J. Heinsohn: Uncertainty and Vagueness in Knowledge Based Systems, John Wiley \& Sons (1994)

[7] M. L. Puri and D. A. Ralescu: Fuzzy random variables; Journal of Mathematical Analysis and its Applications, Vol. 114, pp.409-422 (1986)
[8] E. P. Klement, M. L. Puri and D. A. Ralescu: Law of large numbers and central limit theorem for fuzzy random variables; Cybernetics and Systems Research(R. Trappl, Ed.), North Holland, pp.525-529 (1984)

[9] V. Krätschmer: Integrals of random fuzzy sets: Sociedad de Estaística e Investigación Operativa, Vol. 15, pp.433-469 (2005)

[10] Y. Ogura S. Li and S. V. Kreinovich: Limit Theorems and Applications of Set-Valued and Fuzzy SetValued Random Variables, Kluwer Academic Publishers (2002)

[11] H. Kwakernaak: Fuzzy random variables-II: Algorithms and examples for the discrete case; Information Sciences, Vol. 17, pp.253-278 (1979)

[12] T. Fukuda: Fuzzy random vectors as models for vague but synthetic description of complex random phenomena; IJICIC, Vol. 2, No. 5, pp.1097-1117 (2006)

[13] T. Fukuda: On the set representation for variance of fuzzy random vectors; The 35th SICE Annual Conference, International Session Papers, pp.1363-1368 (1996)

[14] T. Fukuda: On fuzzy random vectors as vague perceptions of random phenomena and their statistical moments; The Proceedings of 35th IEEE CDC, pp.2719-2724 (1996)

[15] T. Fukuda: On variances for a class of fuzzy random vectors; Proceedings of The 36th ISCIE International Symposium on Stochastic Systems Theory and its Applications (2005)

[16] T. Fukuda and Y. Egawa: On fuzzy random vectors and their application to questionnaire survey; The 49th Japan Joint Automatic Control Conference (2006)

[17] Y. Egawa and T. Fukuda: Fuzzy random vectors with stepwise membership levels and their application to questionnaire survey; IJICIC, Vol. 4, No. 1, pp.1-14 (2008)

\section{Author}

Tokuo FukUda (Member)

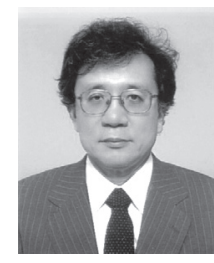

Tokuo FukUDA received the B.S. and M.S. degree in engineering from Kyoto Institute of Technology, Kyoto, Japan in 1975 and 1977 respectively and he received the Ph.D. degree in engineering from Osaka University, Osaka, Japan in 1987. He is now a Professor at Otemon Gakuin University, Osaka, Japan. He is a member of IEEE, ISCIE, SICE, SOFT and Japan SIAM. 